Melanie Grace Y. Cruz, MD

Natividad A. Almazan, MD

Department of Otorhinolaryngology

Head and Neck Surgery

East Avenue Medical Center
Correspondence: Dr. Melanie Grace Y. Cruz Department of Otorhinolaryngology Head and Neck Surgery

6th floor, East Avenue Medical Center

East Avenue, Diliman, Quezon City 1100

Philippines

Phone: (632) 9280611 local 324

Fax: (632) 4356988

Email:eamc_enthns@yahoo.com

melcruzmd@gmail.com

The authors declared that this represents original material that is not being considered for publication or has not been published or accepted for publication elsewhere, in full or in part, in print or electronic media; that the manuscript has been read and approved by all the authors, that the requirements for authorship have been met by each author, and that each author believes that the manuscript represents honest work.

Disclosures: The authors signed disclosures that there are no financial or other (including personal) relationships, intellectual passion, political or religious beliefs, and institutional affiliations that might lead to a conflict of interest.

Presented at the Philippine Society of Otolaryngology Head and Neck Surgery Descriptive Research Contest. September 19, 2013. Natrapharm, The Patriot Building, Parañaque City.

\section{Adult Acute Epiglottitis: An Eight - Year Experience in a Philippine Tertiary Government Hospital}

\begin{abstract}
Objective: To review cases of adult acute epiglottitis in a tertiary government hospital and describe the clinical presentations, diagnostics performed, management and outcomes.

\section{Methods:}

\section{Design: Retrospective Chart Review \\ Setting: $\quad$ Tertiary Government Hospital}

Participants: Records of patients admitted by or referred to the Department of Otolaryngology Head and Neck Surgery with a diagnosis of acute epiglottitis from January 2008 to August 2014 were identified from the department census and charts were retrieved from the Hospital Record Section and evaluated according to inclusion and exclusion criteria. Information regarding demographic data, clinical features, laboratory and other diagnostic examinations, medical management, and length of hospital stay were collected.
\end{abstract}

Results: There were 20 cases in 7 years and 8 months. Most were male, 18 to 37 -years-old, presenting with dysphagia, odynophagia and a swollen epiglottis on laryngoscopy. Abnormal soft-tissue lateral radiographs of the neck and leukocytosis were seen in $73 \%$ and $83 \%$, respectively. Intravenous antibiotics and corticosteroids were administered in all cases, and mean hospital stay was 11.2 days.

Conclusion: Adult acute epiglottitis should be highly suspected in patients presenting with dysphagia, odynophagia, and muffling of the voice even with a normal oropharyngeal examination. History of respiratory infection, co-morbidities, smoking and alcohol intake, concomitant laryngeal pathology and supraglottic structure insults contribute to development of the disease. Laryngoscopy is still the gold standard in diagnosis. Airway protection is mandatory but prophylactic intubation or tracheostomy are not advised. Intravenous antibiotics are necessary and corticosteroids may be of benefit.

Keywords: epiglottitis, supraglottitis, epiglottis, adult, Philippines 
ORIGINAL ARTICLES

Acute epiglottitis is a serious life threatening disease due to its potential for sudden airway obstruction in a previously healthy individual. ${ }^{1,2}$ Structures involved include the supraglottic region of the oropharynx, epiglottis, vallecula, arytenoids and aryepiglottic folds. ${ }^{3}$ Due to the loose and vascular mucosa surrounding the epiglottic area, it is vulnerable to insults caused by inflammation, irritation or allergic reaction. For this reason, the term supraglottitis is more appropriate. ${ }^{4}$

A search of local (HERDIN) and international databases (Embase, PubMed - MEDLINE, Google Scholar, and ScienceDirect) using the following keywords: epiglottitis, supraglottitis, adult, Philippines revealed that this is the second case series on acute epiglottitis in the Philippines with the firststudy done in $1947 .{ }^{5}$ The limited documentation of adult acute epiglottitis in the country and importance of awareness of its urgency prompted this study.

Our objective was to review the cases of adult acute epiglottitis at a tertiary referral government hospital in the Philippines over a span of 7 years and 8 months; and to describe its clinical features, risk factors, diagnostic examinations performed, treatment, and outcomes as measured by improvement of symptoms and length of hospital stay.

\section{METHODS}

This study considered all adult patients diagnosed with supraglottitis - hyperemia and edema of the epiglottis via laryngoscopy, who were admitted by or referred to the Department of Otolaryngology Head and Neck Surgery in a 600-bed tertiary government referral hospital from 2008 to 2014 for inclusion. Those who had supraglotittis due to caustic ingestion were excluded.

Potential cases were identified from the department census and charts were retrieved from the Hospital Record Section and reviewed according to inclusion and exclusion criteria. A total of 20 cases were identified but only 18 charts were available for review. Information regarding demographic data, clinical features, laboratory and other diagnostic examinations, medical management, and length of hospital stay were collected. All data recovered from chart and medical records were included in the analysis. Summary statistics such as mean, median (range) and frequency (proportions) were used to describe continuous ordinal and nominal variables. Data encoding, processing and analysis were performed via Microsoft Excel for Mac 2011 v 14.0.0.

This study was exempted from full review by our hospital IRB and conducted in accordance with the Declaration of Helsinki and the National Guidelines for Biomedical Research of the National Ethics Committee (NEC) of the Philippines. Data collected from the chart review were treated with confidentiality, assigning unique al phanumeric codes in consecutive order to protect the identity of patients. Records were not uploaded on any open databases for public viewing. Attending physicians assigned to the cases were not recorded either.

\section{RESULTS}

Of the 20 consecutive cases of acute epiglottitis identified, 18 charts were available for review. There were 16 males and 4 females, with age range of $18-68$ years old and a median of 33.5 years. One patient who had a recurrence of epiglottitis within the same year was counted twice.

The most common presenting symptoms were dysphagia (100\%), odynophagia (89\%) and voice changes $(83 \%)$ characterized as hoarseness or muffling. (Table 1) One patient who was later intubated additionally presented with irritability, stridor and cyanosis.

Table 1. Presenting symptoms of adult patients with acute supraglotittis $(n=18)$

\begin{tabular}{|l|c|c|c|}
\hline Symptom & $\begin{array}{c}\text { Frequency } \\
(\mathbf{n})\end{array}$ & Total (n) & $\begin{array}{c}\text { Percent } \\
(\%)\end{array}$ \\
\hline Dysphagia & 18 & 18 & 100 \\
\hline Odynophagia & 16 & 18 & 89 \\
\hline Throat Pain* & 6 & 7 & 86 \\
\hline Voice Changes & 15 & 18 & 83 \\
\hline Dyspnea & 10 & 18 & 56 \\
\hline Drooling & 5 & 18 & 28 \\
\hline Irritability & 2 & 18 & 11 \\
\hline Stridor & 2 & 18 & 11 \\
\hline
\end{tabular}

* 11 charts did not indicate presence or absence of the symptom

Hyperemia of the posterior pharyngeal wall and drooling of saliva were observed in half of the cases. Concomitant laryngeal pathologies were observed in 39\% and included cysts, pustules, ulcers and masses. Granulation tissue on the epiglottis was present in the intubated patient and a supraglottic mass was seen in another patient who had a tracheostomy.

Of all the patients, $56 \%$ had previous consults with other physicians and were managed as cases of respiratory tract infection. Other co-morbidities included hypertension (22\%) and diabetes mellitus (22\%). Half were smokers and 13 were alcoholic beverage drinkers. Interestingly, all 8 patients who had a history of fluid intake of extreme temperatures (thermal insult) prior to the onset of symptoms recalled having taken either a cold or hot beverage.

On admission, 78\% had leukocytosis with a mean count of 19.9 $\mathrm{x} 10^{9} / \mathrm{l}$, with predominance of neutrophilis. Chest radiography showed active parenchymal infiltrates in $47 \%$ of the cases and soft-tissue lateral radiographs of the neck were abnormal in $73 \%$ with the following findings: enlarged epiglottis, narrowed airway, and straightening of the vertebra.

Management included intravenous antibiotics (cefuroxime or ceftriaxone) and intravenous hydrocortisone. Ten patients presented 


\section{ORIGINAL ARTICLES}

with dyspnea and half were given oxygen supplementation via nasal cannula. One patient developed severe dyspnea, irritability, cyanosis and stridor and was subsequently intubated. Another patient had a supraglottic mass and eventually needed a tracheostomy.

The time interval from the onset of symptoms to emergency room consult varied from less than 6 hours to as long as two months. The patient with rapid progression of symptoms developed dyspnea in less than 6 hours and was later intubated.

Hospital stay was a median of 8 days (range: 2 - 37 days) for all patients. One patient stayed for 34 days in the hospital due to relapse of epiglottitis within the same admission. Another was admitted for 37 days because of a supraglottic neoplasm, although epiglottic swelling had decreased significantly in this patient by the $16^{\text {th }}$ hospital day. This was the longest time to resolution recorded for significant decrease in epiglottic swelling on laryngoscopy which was noted as early as the second hospital day.

\section{DISCUSSION}

Supraglottitis is an uncommon disease entity that may occur at any age. ${ }^{6}$ Although classically described as a disease of childhood, it is said to have nearly disappeared in children and is now almost exclusively found in adults. ${ }^{7}$

Supraglottitis is traditionally related to Haemophilus influenza infection although its isolation is more common in children (68-72\%) than adults (21-23\%). ${ }^{8}$ Other pathogens have been implicated in adult supraglottitis and may also be caused by thermal injuries suffered from smoking and illicit drug use. ${ }^{1,4}$

Patients in this study were predominantly male (80\%). This was comparable to other series where authors described a $55-81 \%$ preponderance of male subjects. ${ }^{7,-11}$ The ages of the patients in this study ranged from 18-68 years and majority (55\%) belonged to the 18 to 39-year-old age group. This was consistent with studies whose subjects were mostly adults in the second to fourth decade of life. ${ }^{1,710,12}$

The rate of 2.86 cases per year in our study is quite low compared to other single-institution studies reviewed, documenting 3.7-7.1 cases per year., $29-10,13-14$ Interestingly, there was an increase in the number of patients diagnosed with epiglottitis in our institution in 2014 when 7 patients were documented. Some authors suggest that increased awareness of physicians regarding the disease and the greater availability of non-invasive ways of visualizing the laryngeal structures may have contributed to the increased diagnosis rate. ${ }^{1}$

Similar to previous studies, ${ }^{2,6-7,12}$ patients commonly presented in the emergency room with dysphagia, odynophagia, and voice changes.
Throat pain was also present in 6 out of 7 patients who were asked about this symptom.

Nine of the patients initially seen by other physicians were diagnosed as simple cases of URTI. This may be attributed to laryngoscopy not being a part of routine examination in the general setting. In addition, patients with supraglottitis may also present with a normalappearing oropharynx, which may mistakenly preclude the need for further examination. The abovementioned factors may have possibly resulted in misdiagnosis of cases; other studies cite $67 \%$ to $71 \%$ of cases were commonly assessed to be URTI, pneumonia, and angina with pharyngitis. Some patients may even require intubation on follow up. $^{2,9}$

Adult supraglottitis has a more variable presentation depending on etiology, and the rates at which symptoms appear help prognosticate its outcome. If patients complain primarily of sore throat and dysphagia with a more gradual onset, which was as long as a two months in this study, it may be more likely have a milder course. ${ }^{1}$ In contrast, patients who present with dyspnea, stridor, drooling and isolation of $H$. influenza are more likely to have airway complaints and a more aggressive course of disease. ${ }^{1}$ The patient who presented with a sudden onset of dyspnea, dysphagia, odynophagia, drooling, and stridor 6 hours prior to consult was immediately intubated in the emergency room.

A thorough history and physical examination is vital to diagnosis. Comorbidities such as hypertension, diabetes, alcoholism, smoking history and a recent upper respiratory infection that may be elicited in the history may play a role in the course of the disease, as was also found in patients in this study. ${ }^{1,6,10,14}$ Other findings may include epiglottic abscesses, history of nasopharyngeal carcinoma, tonsillitis, and vallecular cysts. ${ }^{1,2,4,6,10,14}$ In this study, $39 \%$ of patients showed any of the following supraglottic findings on laryngoscopy: pustules, cyst and granulation tissue on the epiglottis and hypopharyngeal mass.

Abnormal chest radiographs in 8 out of 17 patients had active parenchymal infiltrates which may be attributed to pneumonia, empyema or pulmonary edema. ${ }^{1,49}$

Soft tissue lateral radiographs of the neck were positive in $73 \%$ of the cases. This was consistent with other studies which yielded a positive result in $68-89 \%$ of cases. ${ }^{1,6,9,10,14}$ Swelling of the epiglottis, classically described as the "thumb sign" may be found in as many as $71 \%$ of patients. ${ }^{5}$ Laryngoscopy is still the gold standard in diagnosis and should be done in all cases if possible. It is important to note that laryngoscopy did not precipitate airway obstruction in any of the patients in this study. This was consistent with the findings of Mayo-Smith et al. wherein none of the 364 patients seen over 18 years developed airway obstruction 
secondary to laryngoscopy. ${ }^{1}$ Leukocytosis was seen in $83 \%$ of the cases. This was slightly lower compared to studies showing leukocytosis in 91$100 \%$ of patients. ${ }^{2,8,11,14}$ Although the test is sensitive, blood counts are not known to be helpful in diagnosing acute supraglottitis. ${ }^{15}$

The presence of concomitant laryngeal pathology (17\%), a history of thermal insult to the supraglottic structures (22\%), with $22 \%$ having both conditions is suggestive that non-infectious external factors may contribute to the development of supraglottitis.

The most important aspect of management is airway protection. In this study, 5 patients were given oxygen supplementation via nasal cannula, one was intubated and one other underwent tracheostomy. Prophylactic intubation may be unnecessary in the absence of warning signs of airway compromise. Sack and Brock emphasized that the key to management is awareness of the disease and close monitoring of the airway. ${ }^{16}$ In the presence of more than $50 \%$ narrowing, intubation may be performed depending on the severity of the patient's condition. ${ }^{11}$ Therefore, instead of routine prophylactic intubation, management should be focused on the patient's needs and condition. ${ }^{9}$

Antibiotic treatment is also necessary and should cover the most common etiologic agents. Cephalosporins such as ceftriaxone, cefuroxime, and cefotaxime have been used. ${ }^{7,9}$ All patients in our study were started on intravenous cefuroxime or ceftriaxone, and intravenous hydrocortisone. Corticosteroids have been used prophylactically, with conflicting findings in the literature. Some claim their use has not been found to provide reduction in the need for intubation, nor affect the total length of hospital stay., ${ }^{1,2}$ However, the limited number of patients in their study did not allow for a firmer conclusion regarding corticosteroid use. ${ }^{1,2}$

It is advocated that patients be admitted to the ICU for close monitoring. ${ }^{3}$ However, if this is not feasible, patients may be admitted to the ward provided they are stable with an adequate airway and are being managed with antibiotics and corticosteroids. Patients in this study were observed in the ward for a median of 8 days (2-37 days). Personnel equipped with the ability to intubate or perform tracheostomy must always be available. Repeated attempts at intubation should be avoided as it may increase periepiglottic swelling causing more morbidity. ${ }^{16}$

In summary, adult acute epiglottitis should be suspected in patients who present with progressive dysphagia, odynophagia, voice changes, and throat pain especially when there is a normal oropharyngeal examination. Leukocytosis and positive soft tissue lateral radiograph of the neck may support its diagnosis and may warrant the need for laryngoscopy for confirmation. Airway protection is mandatory but prophylactic intubation or tracheostomy are not advised. Intravenous antibiotics are necessary and corticosteroids may be of benefit.

The limitations of the study include a small sample size and few correlations and analyses on the other unusual factors that can also have effects on the development of the condition such as concomitant laryngeal pathology, thermal injuries, smoking and alcoholism.

We recommend a larger-scale, multicenter prevalence study of adult acute epiglottitis. Studies concerning other factors that may be relevant in the clinical course of the disease such as concomitant laryngeal pathology, recent thermal injury to the supraglottic region, smoking, alcoholism, and hyperemia of the pharynx are also suggested.

\section{REFERENCES}

1. Mayo-Smith MF, Spinale JW, Donskey CJ, Yukawa M, Li RH, Schiffman FJ. Acute epiglottitis. An 18-year experience in Rhode Island. Chest. 1995 Dec;108(6):1640-7. PubMed PMID: 7497775.

2. Wick F, Ballmer PE, Haller A. Acute epiglottitis in adults. Swiss Med Wkly. 2002 Oct 12; 132(37-38): 541-7. PubMed PMID: 12557859.

3. Gompf SG, Bowman JG. Epiglottitis. Medscape. 2013 Apr 13 [cited 2013 Jun 17]. Available from: http://emedicine.medscape.com/article/763612-overview

4. Rivers RL. Acute epiglottitis (supraglottitis). J Forensic Sci. 1979 Apr;24(2): 470-2

5. Yambao CV. Acute epiglottitis. J Philipp Med Assoc. 1947 Feb; 23(2):47-9. PubMed PMID: 20254008.

6. $\mathrm{Ng} \mathrm{HL}$, Sin LM, Li MF, Que TL, Anandaciva S. Acute epiglottitis in adults: a retrospective review of 106 patients in Hong Kong. Emerg Med J. 2008 May;25(5): 253-5. DOI: 10.1136/emj.2007.050153. PubMed PMID: 18434453.

7. Briem B, Thorvardsson O, Petersen H. Acute epiglottitis in Iceland 1983-2005. Auris Nasus Larynx. 2009 Feb; 36(1):46-52. DOI: 10.1016/j.anl.2008.03.012. PubMed PMID: 18502071

8. Berg S, Trollfors B, Nylen O, Hugosson S, Prellner K, Carenfelt C. Incidence, aetiology, and prognosis of acute epiglottitis in children and adults in Sweden. Scand J Infect Dis. 1996;28(3):261-4. PubMed PMID: 8863357.

9. Sheikh KH, Mostow SR. Epiglottitis-an increasing problem for adults. West J Med. 1989 Nov 151(5):520-524. PubMed PMID: 2603419 PubMed Central PMCID: PMC1026785.

10. Sarkar S, Roychoudhury A, Roychoudhury BK, Acute epiglottitis in adults - a recent review in an Indian hospital. Indian J Otolaryngol Head Neck Surg. 2009 Sep; 61(3):197-199. PubMed PMID: 23120634. PubMed Central PMCID: PMC3449988.

11. Qazi IM, Jafar AM, Hadi KA, Hussain Z. Acute epiglottitis: a retrospective review of 47 patients in Kuwait. Indian J Otolaryngol Head Neck Surg. 2009 Dec; 61(4):301-305. DOI: 10.1007/s12070 009-0087-4. PubMed PMID: 23120655. PubMed Central PMCID: PMC3450074.

12. Bizaki AJ, Numminen J, Vasama JP, Laranne J, Rautiainen M. Acute supraglottitis in adults in Finland: review and analysis of 308 cases. Laryngoscope. 2011 Oct; 121(10):2107-13. DOI: 10.1002/lary.22147. PubMed PMID: 21898436.

13. Cheung $\mathrm{CH}$. Case and literature review: adult acute epiglottitis - rising incidence or increasing awareness? Hong Kong J Emerg Med. 2001 Oct; 8(4): 227- 231.

14. Lon SA, Lateef M, Sajad M. Acute epiglottitis: A review of 50 patients. Indian J Otolaryngol Head Neck Surg. 2006 Apr; 58(2):178-80. DOI: 10.1007/BF03050781. PubMed PMID: 23120278. PubMed Central PMCID: PMC3450762.

15. Al-Qudah M, Shetty S, Alomari M, Alqdah M. Acute adult supraglottitis: current management and treatment. South Med J. 2010 Aug;103(8):800-4. DOI: 10.1097/SMJ.0b013e3181e538d8. PubMed PMID: 20622745.

16. Sack JL, Brock CD. Identifying acute epiglottitis in adults. High degree of awareness, close monitoring are key. Postgrad Med. 2002 Jul; 112 (1):81-2, 85-6. PubMed PMID: 12146095. 